\title{
Palm Vein Recognition Algorithm using Multilobe Differential Filters
}

\author{
E. I. Safronova ${ }^{1}$, E. A. Pavelyeva ${ }^{1}$ \\ katyasafit@gmail.com|paveljeva@yandex.ru \\ ${ }^{1}$ Faculty of Computational Mathematics and Cybernetics, Lomonosov Moscow State University, Moscow, Russia

\begin{abstract}
In this article the new algorithm for palm vein recognition using multilobe differential filters is proposed. After palm vein image preprocessing vein structure is detected based on principal curvatures. The image is considered as a surface in a three-dimensional space. Some vein points are selected using the maximum principal curvature values, and the other vein points are found from starting points by moving along the direction of minimum principal curvature. Multilobe differential filters are used to extract feature maps for vein images. These filters are flexible in terms of basic lobe choice and spatial configuration of lobes. The multilobe differential filters used in the article simulate vein branch points, and Gaussian kernel is used as the basic lobe. The normalized root-mean-square error is applied for image matching. Experimental results using CASIA multi-spectral palmprint image database demonstrate the effectiveness of the proposed method. The value of EER=0.01693 is obtained.
\end{abstract}

Keywords: biometrics, palm vein recognition, principal curvatures, multilobe differential filters

\section{Introduction}

Biometrics recognizes individuals based on the characteristics, known as modalities, that uniquely identify a person from an entire population based on intrinsic physical or behavioral traits [8]. Each biometric modality has its advantages.

Palm vein recognition is an emerging personal identification technology. Since veins are usually not visible to others, they can be used for personal identification purposes at low risk of forgery or theft. Veins are captured by infrared camera since deoxygenated hemoglobin in the vein blood absorbs near infrared light. Among other important advantages vein patterns are quite unique to the owners, image acquisition does not require physical contact, and the system can be made compact. Extracting vein structure at their accurate positions regardless of their thickness and brightness is necessary for accurate personal identification.

Ordinarily, palm vein recognition algorithm consists of three steps (Fig. 1). The first one, region of interest (ROI) extraction, includes properly extraction and image preprocessing.

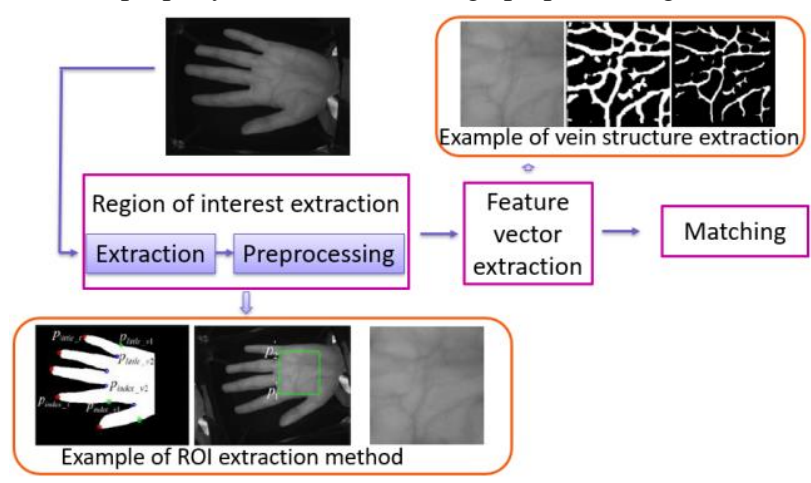

Fig. 1. The scheme of palm vein recognition algorithm.

The second step, feature vector extraction, represents the main difference between existing approaches. Some methods could be derived from other biometric recognition algorithms: for example, methods that employ statistical information (such as local binary patterns, local derivative patterns) [12, 21], methods that extract feature points and then match them $[11,14$, 26], subspace-based methods [25], phase based methods [7, 17, 18 ] and etc. Some approaches were developed specifically for vein recognition $[5,15]$.

The last algorithm step, image matching, is based on feature vector type. At this stage the distance between palm vein images is calculated.

Much recent work has been focused on employing deep convolutional neural networks in various domains, and the field of biometrics is not an exception. Consider some examples of applying deep learning methods to different steps of palm vein recognition algorithm. To perform the first algorithm step and to extract the palm vein ROI's, the region-based convolution neural network that localize the region of interest might be used. The reference article [9] gives an example of successful realization. Deep convolution neural network could be trained as feature extractor. Authors of [25] proposed to use the pre-trained DCNN model trained on large-scale database like ImageNet. They described a novel global max-pooling of preserving spatial position information that is applied for the feature maps of convolutional layer to localize vein minutiae features. As for matching, the last step of recognition algorithm, we can consider [24]. The proposed biometric authentication approach is based on Siamese convolution neural network framework with a triplet loss function, that enables an idea to learn the distance metric between positive, anchor, and negative embeddings.

In this article we propose the use of multilobe differential filters (MLDF) for palm vein recognition. Recently, ordinal measures have achieved promising performance in different biometric tasks [4, 22, 23]. Ordinal measures represent a general concept of image analysis with numerous variants of different parameter settings such as location, scale, orientation etc. They are defined as the relative ordering of a set of regional image features (e.g. average intensity, Gabor wavelet coefficients, etc.). The main idea of ordinal measures is to distinguish the image structures with specific texture. MLDF can represent ordinal features. MLDF is a general concept of differential and bandpass filters and is flexible in terms of basic lobe choice, spatial configuration of lobes, etc. [22]. In this article the positive and negative lobes are represented by Gaussian filters.

The rest of this paper is organized as follows: in Section 2 the vein image segmentation and ROI extraction algorithms are described. Our ROI image preprocessing approach is given in Section 3. The main steps of the proposed vein structure extraction algorithm are introduced in Section 4. Feature extraction and feature matching methods are presented in Sections 5 and 6, respectively. The experimental results for images from CASIA multi-spectral palmprint image database [3] are given in Section 7. Finally, Section 8 concludes this paper.

\section{Region of interest extraction}

The proposed palm vein ROI segmentation scheme is illustrated in Fig. 2. Gaussian blur is first adopted for smoothing the images (Fig. 2 a). Then OTSU algorithm [16] is applied to extract hand contours from grayscale palm vein images (Fig. 2 b). Since the lighting provides poor contour segmentation performance at the wrist section of the image [10], the 
coordinates of the centroid $C\left(X_{c}, Y_{c}\right)$ of the binary image are calculated:

$$
X_{c}=\frac{1}{|O|} \sum_{i \in O} X_{i}, Y_{c}=\frac{1}{|O|} \sum_{i \in O} Y_{i},
$$

where $O$ is the set of object point on the binary image and $|O|$ is the power of this set. Then the lower part of image close to wrist is filled line by line with zeros until the distance from the filled area to $C$ is equal to $L=125$. (Fig. 2 c). Taking the midpoint of upper line of the filled area as the reference point $W$, the Euclidean distances between $W$ and all points on the hand contour are calculated to obtain the radial distance function (RDF), as shown in Fig. 2(e). We can see that the minima in the $\mathrm{RDF}$ correspond to the finger valleys of the final binary image. The points between index and middle fingers, $P_{1}$ (Fig. $2 \mathrm{~d}$ ), and forth and little fingers, $P_{2}$, can be taken as landmarks for extraction of square ROI [13].

To eliminate the influence of palm rotation, the image is rotated to the angle $\theta$ which is the angle between the line $P_{1} P_{2}$ and the horizontal line:

$$
\theta=\operatorname{arctg}\left(\left(Y_{P_{1}}-Y_{P_{2}}\right) /\left(X_{P_{1}}-Z_{P_{2}}\right)\right),
$$

where $\left(X_{P_{1}}, Y_{P_{1}}\right)$ and $\left(X_{P_{2}}, Y_{P_{2}}\right)$ are the coordinates of $P_{1}$ and $P_{2}$, respectively.

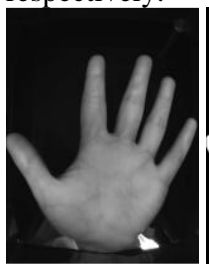

(a)

(e)

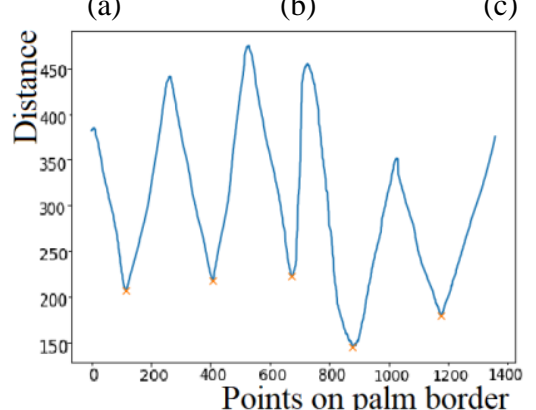

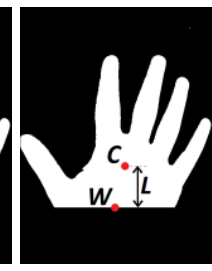

(c)

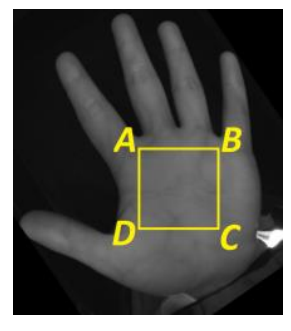

(f)
Fig. 2. Illustration of palm vein ROI extraction: (a) original palm image, (b) binary hand image, (c) reference point $W$ and centroid $C$, (d) two landmark points $P_{1}$ and $P_{2}$, (e) RDF, (f) the square region of interest $A B C D$ on the rotated image.

We denote the distance between $P_{1}$ and $P_{2}$ as $d$. Then the square region $A B C D$ with side $d$ is extracted (Fig. $2 \mathrm{f}$ ), where the top side $A B$ is lower by $d / 6$ and parallel to $P_{1} P_{2}$ [7]. Then $A B C D$ is rescaled by bilinear interpolation to the square size $128 \times 128$ which is called as ROI.

To reduce the non-uniform illumination appearing in palm vein images the background brightness is found as convolution of ROI image with box filter size $17 \times 17$. Then the background is subtracted and the histogram is stretched (Fig. 3).

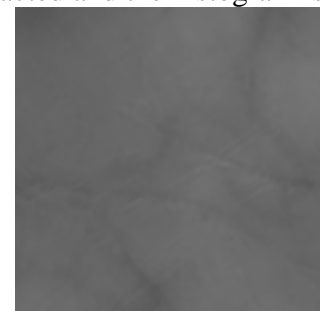

(a)

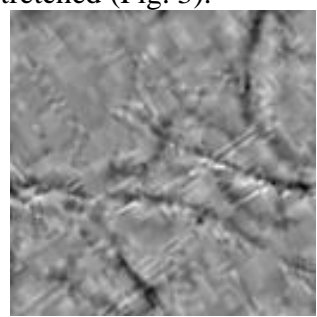

(b)
Fig. 3. (a) original ROI image, (b) ROI image with uniform illumination.

\section{Image preprocessing}

The following scheme of palm vein images preprocessing is used to emphasize veins structure (Fig. 4).

At first, contrast-limited adaptive histogram equalization (CLAHE) technique [29] is used to improve contrast of images. After contrast enhancement all image details including noise and glares are sharper. In order to smooth the undesirable details, non-local means (NLM) algorithm [2] is used to reduce noise. NLM smoothies also veins a little so CLAHE is applied again to obtain distinguishable veins.

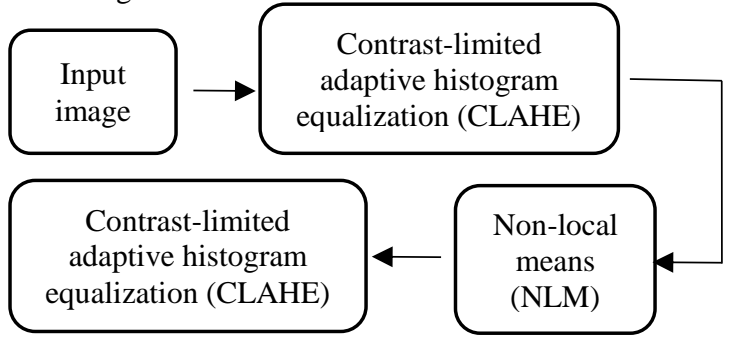

Fig. 4. The scheme of vein image preprocessing algorithm.

Fig. 5 shows the ROI of palm vein image and the results of preprocessing algorithm. After preprocessing veins become sharper and more distinguishable.

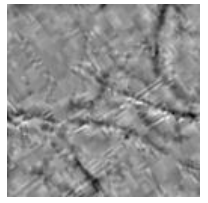

(a)

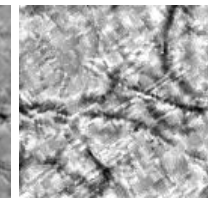

(b)

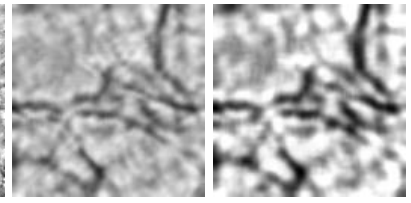

(c) (d)
Fig. 5. The results of image preprocessing: (a) ROI, (b) ROI after the first step of preprocessing - CLAHE, (c) ROI after the second step of preprocessing - NLM, (d) ROI after the third step of preprocessing - CLAHE.

\section{Vein structure extraction}

The next step is vein structure extraction. Consider an image as a surface in a three-dimensional space, where the brightness value of the pixels is the $z$-coordinate. We are going to calculate the values of the principal curvatures and principal directions at each point by the following algorithm.

Let $L(x, y)$ denote the image intensity field at the pixel position. The gradient $G(x, y)$, which is a vector field of $L(x, y)$, is defined as follows:

$$
G(x, y)=\left(\frac{\partial L(x, y)}{\partial x}, \frac{\partial L(x, y)}{\partial y}\right) .
$$

Then the normalized gradient after hard thresholding is defined as:

$$
G_{\gamma}(x, y)=\left\{\begin{array}{c}
\frac{G(x, y)}{\|G(x, y)\|}, \quad\|G(x, y)\| \geq \gamma, \\
0, \quad \| G(x, y \|<\gamma
\end{array},\right.
$$

where $\gamma$ is a threshold level. In the experiments we use $\gamma=4$. The normalized gradient field contains noisy components so we smooth it. Let $G_{\gamma}(x, y)=\left(g_{x}(x, y), g_{y}(x, y)\right)$. Then smoothed normalized gradient components, $h_{x}$ and $h_{y}$, are defined as: $h_{x}(x, y)=g_{x}(x, y) * H(x, y), h_{y}(x, y)=g_{y}(x, y) * H(x, y)$, where $H(x, y)=\frac{1}{2 \pi \sigma^{2}} e^{-\frac{x^{2}+y^{2}}{2 \sigma^{2}}}$ is a Gaussian function.

The local shape characteristics of an image $L(x, y)$ at a point $(x, y)$ can be described by the Hessian matrix

$$
H_{S}(x, y)=\left(\begin{array}{ll}
\frac{\partial h_{x}(x, y)}{\partial x} & \frac{\partial h_{x}(x, y)}{\partial y} \\
\frac{\partial h_{y}(x, y)}{\partial x} & \frac{\partial h_{y}(x, y)}{\partial y}
\end{array}\right) .
$$


Let $\lambda_{1}, \lambda_{2}$ be the eigenvalues and $v_{1}, v_{2}$ be the corresponding eigenvectors of $H_{S}(x, y),\left|\lambda_{1}\right|>\left|\lambda_{2}\right|$. Then the two principal directions, the directions of the maximum and minimum curvature, are determined by two eigenvectors, $v_{1}$, $v_{2}$. Consequently, two eigenvalues $\lambda_{1}, \lambda_{2}$ represent the principal curvatures (the curvatures along the principal directions) [5] (Fig. 6).

In order to catch veins of different widths, consider the set of parameters $\sigma$ for the Gauss function: $\sigma_{0}, \ldots, \sigma_{n-1}$, where $n=10$, $\sigma_{i}=\sigma_{0} \cdot \sqrt[4]{2^{i}}, \sigma_{0}=2, i=0,1, \ldots, 9$. For each value of $\sigma$ the Hessian matrix is constructed, at each point the maximum positive eigenvalue $\lambda_{1}$ and an eigenvector $v_{2}$ of the minimum eigenvalue are calculated. Then, at each point of the image, the largest value of $\lambda_{1}$ over all $\sigma$ and the corresponding vector $v_{2}$ are taken.

In Fig. 6 the surface with a tubular shape like veins in infrared images is shown. At a point $(x, y), \lambda_{1}, \lambda_{2}$ represent the maximum and the minimum principal curvature, vectors $v_{1}, v_{2}$ - the direction of them. Note that tubular-shaped regions have maximum principal curvature $\lambda_{1}$ higher than other regions. Next, mention that vector $v_{1}$ is directed across tubular direction and vector $v_{2}$-along tubular direction [20] (Fig. 7). According to this we select points with highest maximum principal curvature values as points that certainly belong to veins (Fig. 8 b). So, the other vein points can be found from starting points by moving along direction of vector $v_{2}$ by $\left|\lambda_{1}\right|$. The extracted vein structures are shown in Fig. 8.

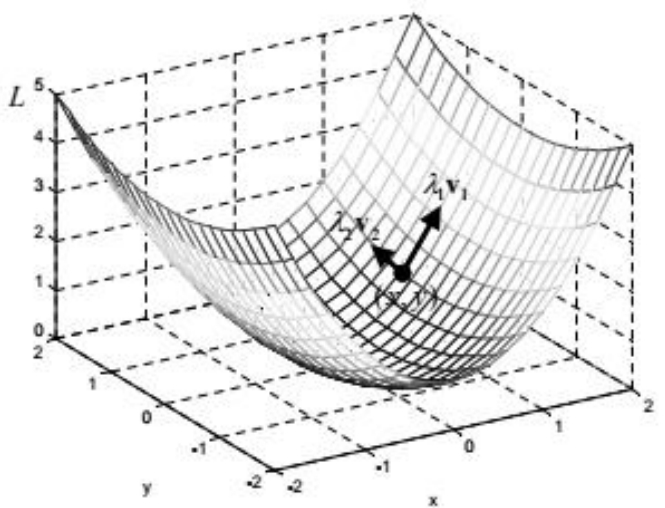

Fig. 6. Concept of the principal direction and the principal curvature: $\lambda_{1}, \lambda_{2}$ represent the maximum and the minimum principal curvature, $v_{1}, v_{2}$ - the direction of them.

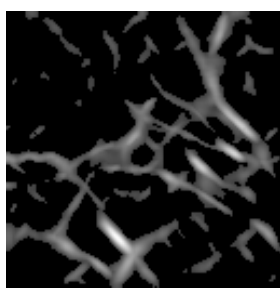

(a)

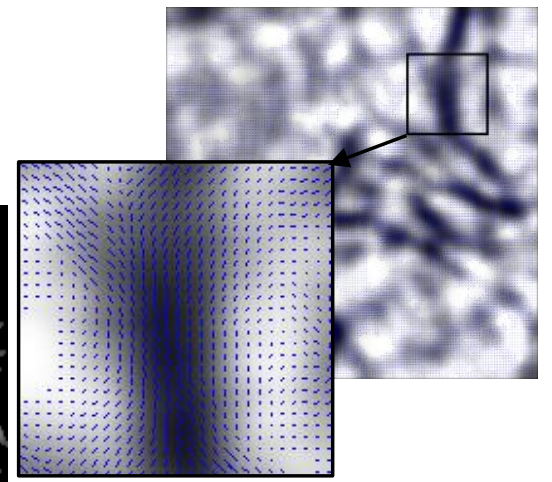

(b)
Fig. 7. (a) The computed positive maximum principal curvatures, $\lambda_{1}$, of ROI image (Fig. 5d), (b) Vector field of direction for the minimum principal curvature $v_{2}$ with length in proportion to $\lambda_{1}$.
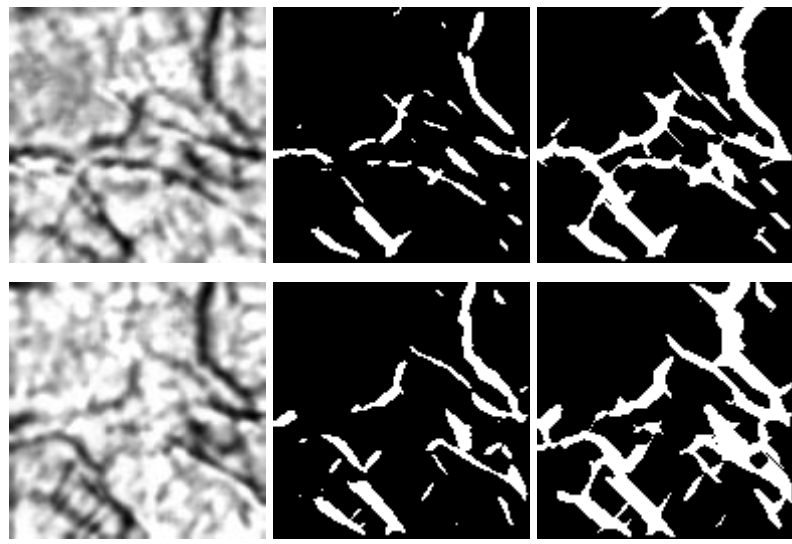

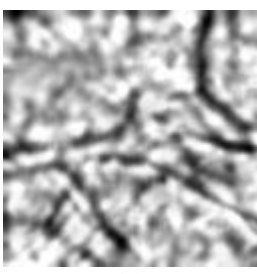

(a)

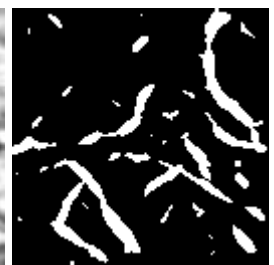

(b)

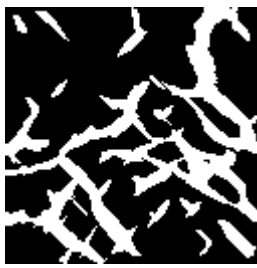

(c)

Fig. 8. Vein structures extraction: (a) ROI for different palm vein images of one person, (b) starting points with highest $\lambda_{1}$ belonging to veins, (c) found vein structure.

\section{Feature extraction}

The palm vein structure images after previous stage are not accurate enough so we will use them as a mask. We invert preprocessed ROI images so that vein pixels have higher values than background pixels and apply masks (Fig. 9).

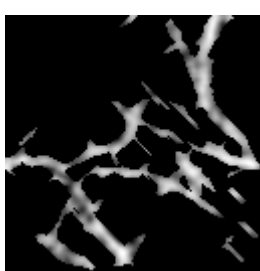

Fig. 9. The mask of ROI for different palm vein images of one person.

In this paper we propose the use of multilobe differential filters (MLDF) [22] for palm vein feature extraction, aiming to highlight vein branch points. We use the Gaussian kernel as the basic lobe, so mathematically the MLDFs are given as follows:

$$
M L D F=C_{p} \sum_{i=1}^{N_{p}} \frac{1}{\sqrt{2 \pi \sigma_{p i}}} e^{\frac{-\left(X-\mu_{p i}\right)^{2}}{2 \sigma_{p i}^{2}}}-C_{n} \sum_{i=1}^{N_{n}} \frac{1}{\sqrt{2 \pi \sigma_{n i}}},
$$

where the variables $\mu$ and $\sigma$ denote the central positions and the scales of a $2 \mathrm{D}$ Gaussian filters respectively, $N_{p}$ denote the number of positive lobes, and $N_{n}$ denote the number of negative lobes. Constant coefficients $C_{p}$ and $C_{n}$ are used to ensure zero sum of the MLDF.

The most compelling feature of MLDF compared with traditional differential filters is that it decouples the settings of intralobe (scale) and interlobe (distance) parameters. Some examples of MLDF with different settings of parameters (distance, scale, orientation, number, and location) are illustrated in Fig. 10 a. MLDF can have visual meaning, for example, MLDF can represent point, line, edge, ridge, corner, slope (Fig. 10 b) [22]. 


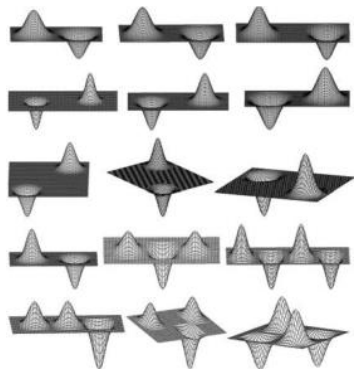

(a)
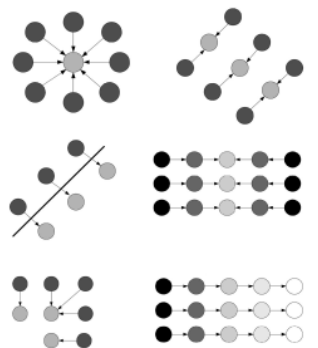

(b)
Fig. 10. (a) Some examples of multilobe differential filters. (b) Visual meanings of MLDF.

In order to select the points of interest, consider MLDF that simulate vein branch points (Fig. 11). We take the convolution results of the ROI images with the proposed MLDF kernels to obtain the feature maps of vein images. Compared with the previous approach [22], the convolution results are not binarized. It enables us to use more information and to achieve higher accuracy.

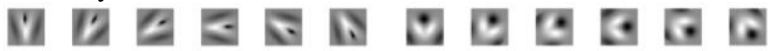 \\ U IV B E S I S G G G a

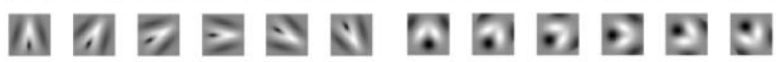

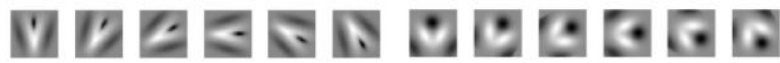 4

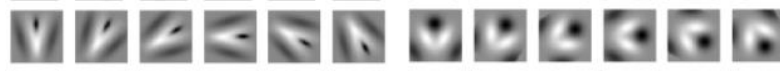

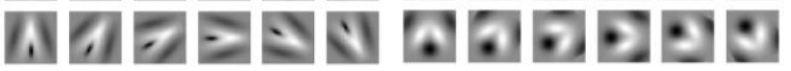

(a)

(b)

Fig. 11. Illustration of proposed multilobe differential filters for vein image analysis: (a) the first group: with 30 degree angle between two positive lobes; (b) the second group: with 60 degree angle between two positive lobes.

\section{Feature matching}

In order to provide slight translation and rotation invariance matching, the normalized root-mean-square error (NRMSE) is proposed for feature maps matching [6]:

$$
\begin{aligned}
& E(f(x, y), g(x, y))^{2} \\
& =\min _{\alpha, x_{0}, y_{0}} \frac{\sum_{x, y}\left|\alpha g\left(x-x_{0}, y-y_{0}\right)-f(x, y)\right|^{2}}{\sum_{x, y}|f(x, y)|^{2}} \\
& =1-\frac{\max _{x_{0}, y_{0}}\left|r_{f g}\left(x_{0}, y_{0}\right)\right|^{2}}{\sum_{x, y}|f(x, y)|^{2} \sum_{x, y}|g(x, y)|^{2}},
\end{aligned}
$$

where the summation is over all the pixels $(x, y)$ in the image;

$$
\begin{aligned}
r_{f g}\left(x_{0}, y_{0}\right)=\sum_{x, y} f(x, y) g^{*}\left(x-x_{0}, y-y_{0}\right) \\
=\sum_{u, v} F(u, v) G^{*}(u, v) e^{i 2 \pi\left(\frac{u x_{0}}{M}+\frac{v y_{0}}{N}\right)}
\end{aligned}
$$

is the cross correlation of $f(x, y)$ and $g(x, y) ; N$ and $M$ are the image dimensions; $*$ denotes complex conjugation; uppercase letters represent the DFT of their lowercase counterparts, as given by the relation

$$
F(u, v)=\sum_{x, y} \frac{f(x, y)}{\sqrt{M N}} e^{-i 2 \pi\left(\frac{u x}{M}+\frac{v y}{N}\right)} .
$$

\section{Experimental results}

In our research CASIA Multi-Spectral Palmprint Image Database [3] was used. It contains 7200 palm images captured from 100 different people using a self-designed multiple spectral imaging device. Each sample contains six palm images which are captured at the same time with six different electromagnetic spectrums.

Given the intra and interclass vein matching results, the recognition performance is measured by the following indicators.

1) False Acceptance rate (FAR): the probability that the system incorrectly matches the input pattern to a non-matching template in the database.

2) False Reject Rate (FRR): the probability that the system fails to detect a match between the input pattern and a matching template in the database.

3) Equal error rate (EER), i.e., the cross-over error rate when FAR is equal to the FRR. Lower EER means higher accuracy of a biometric matcher.

4) The distribution of genuine and impostor scores.

In our study the part of the images from CASIA database obtained at $850 \mathrm{~nm}$ are taken. In Fig. 12 the results of the proposed algorithm are presented. We applied the first MLDF kernels group (Fig. 11 a) and both MLDF kernels group (Fig. $11 \mathrm{a}, \mathrm{b}$ ). The EER results are 0.01693 in the first case and 0.01862 in the second case.

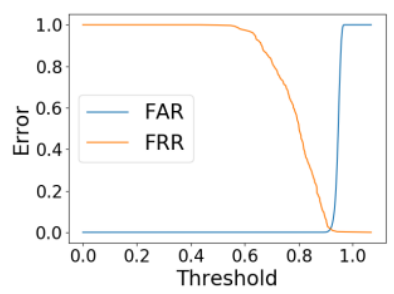

(a)

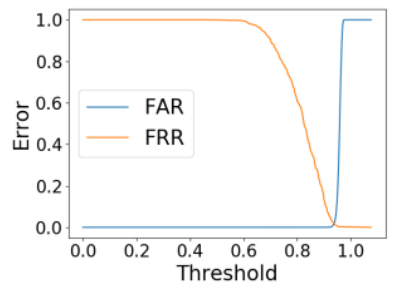

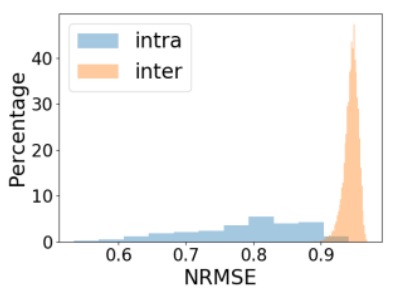

(b)

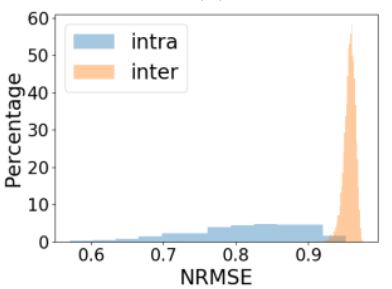

Fig. 12. Illustrations of the distribution of genuine and impostor scores, FAR and FRR curves: (a), (c) FAR and FRR curves; (b), (d) the distribution of genuine and impostor scores. The top row $(a-b)$ corresponds to the first MLDF kernels group (Fig. $10 \mathrm{a}$ ), the bottom row (c-d) corresponds to both MLDF kernels group (Fig. $10 \mathrm{a}, \mathrm{b}$ ).

A comparison of EERs derived from different approaches for CASIA database is given in Table 1. It shows the good performance of the proposed method. As an improvement of the proposed method, multilobe differential filters selection using machine learning techniques can be applied [23].

\begin{tabular}{|l|l|l|}
\hline \multicolumn{1}{|c|}{ Reference } & \multicolumn{1}{c|}{ Method } & \multicolumn{1}{c|}{ EER } \\
\hline Kang W., Wu Q [10] & $\begin{array}{l}\text { Improved } \\
\text { LBP }\end{array}$ & 0.00267 \\
\hline Zhou Y., Kumar A. [28] & NMRT & 0.0051 \\
\cline { 2 - 3 } & Hessian phase & 0.0144 \\
\hline $\begin{array}{l}\text { Thapar D., Jaswal G., } \\
\text { Nigam A., Kanhangad V. } \\
\text { [24] }\end{array}$ & PVSNet & 0.0371 \\
\hline $\begin{array}{l}\text { Bhilare S., Jaswal G., } \\
\text { Kanhangad V., Nigam A. } \\
\text { [1] }\end{array}$ & $\begin{array}{l}\text { Deep } \\
\text { matching }\end{array}$ & 0.0261 \\
\hline $\begin{array}{l}\text { Raghavendra R., Busch } \\
\text { C. [19] }\end{array}$ & $\begin{array}{l}\text { LD-KDA- } \\
\text { SRC }\end{array}$ & $0.1010 \pm 0.0102$ \\
\hline Proposed method & MLDF & 0.01693 \\
\hline
\end{tabular}

Table 1. Summary of related approaches for palm vein verification using CASIA database. 


\section{Conclusion}

In this paper the new palm vein recognition method based on multilobe differential filters is proposed. Palm vein image preprocessing and palm vein structure extraction are described. The points with high maximum principal curvature values are selected as starting points, and the other vein points are calculated using the direction of minimum principal curvature. Feature extraction is based on MLDF where the Gaussian kernel is used as the basic lobe. NRMSE is used for image matching. Experimental results demonstrate that the proposed approach gives good recognition accuracy.

\section{References}

[1] Bhilare S., Jaswal G., Kanhangad V., Nigam A. Singlesensor hand-vein multimodal biometric recognition using multiscale deep pyramidal approach //Machine Vision and Applications. - 2018. - Vol. 29. - №. 8. - Pp. 1269-1286.

[2] Buades A., Coll B., Morel J. M. A non-local algorithm for image denoising //Computer Vision and Pattern Recognition, 2005. CVPR 2005. IEEE Computer Society Conference on. - IEEE, 2005. - Vol. 2. - Pp. 60-65.

[3] CASIA Multi-Spectral Palmprint Image Database http://biometrics.idealtest.org/

[4] Chai Z., Sun Z., Mendez-Vazquez H., He R., Tan T. Gabor ordinal measures for face recognition //IEEE transactions on information forensics and security. - 2013. - Vol. 9. - №. 1. - Pp. 14-26.

[5] Choi J. H., Song W., Kim T., Lee S. R., Kim H. T. Finger vein extraction using gradient normalization and principal curvature //Image Processing: Machine Vision Applications II. - International Society for Optics and Photonics, 2009. Vol. 7251. - Pp. 725111.

[6] Fienup J. R. Invariant error metrics for image reconstruction //Applied optics. - 1997. - Vol. 36. - №. 32. - Pp. 83528357.

[7] Han W. Y., Lee J. C. Palm vein recognition using adaptive Gabor filter //Expert Systems with Applications. - 2012. Vol. 39. - №. 18. - Pp. 13225-13234.

[8] Jain A. K., Bolle R., Pankanti S. (ed.). Biometrics: personal identification in networked society. - Springer Science \& Business Media, 2006. - Vol. 479.

[9] Jha R. R., Thapar D., Patil S. M., Nigam A. Ubsegnet: Unified biometric region of interest segmentation network - IEEE, 2017.

[10] Kang W., Wu Q. Contactless palm vein recognition using a mutual foreground-based local binary pattern //IEEE Transactions on information forensics and security. -2014. - Vol. 9. - №. 11. - Pp. 1974-1985.

[11] Ladoux P. O., Rosenberger C., Dorizzi B. Palm vein verification system based on SIFT matching //International Conference on Biometrics. - Springer, Berlin, Heidelberg, 2009. - Pp. 1290-1298.

[12] Lee E. C., Jung H., Kim D. New finger biometric method using near infrared imaging //Sensors. - 2011. - Vol. 11. №. 3. - Pp. 2319-2333.

[13] Lin C. L., Chuang T. C., Fan K. C. Palmprint verification using hierarchical decomposition //Pattern Recognition. 2005. - Vol. 38. - №. 12. - Pp. 2639-2652.

[14] Matsuda Y., Miura N., Nagasaka A., Kiyomizu H., Miyatake T. Finger-vein authentication based on deformation-tolerant feature-point matching //Machine Vision and Applications. - 2016. - Vol. 27. - №. 2. - Pp. 237-250.

[15] Miura N., Nagasaka A., Miyatake T. Feature extraction of finger-vein patterns based on repeated line tracking and its application to personal identification //Machine vision and applications. - 2004. - Vol. 15. - №. 4. - Pp. 194-203.
[16] Otsu N. A threshold selection method from gray-level histograms //IEEE transactions on systems, man, and cybernetics. - 1979. - Vol. 9. - №. 1. - Pp. 62-66.

[17] Pavelyeva, E. A. Image processing and analysis based on the use of phase information //Computer Optics. - 2018. Vol. 42. - № 6. - Pp. 1022-1034.

[18] Protsenko M. A., Pavelyeva E. A. Iris image key points descriptors based on phase congruency //ISPRS International Archives of the Photogrammetry, Remote Sensing and Spatial Information Sciences. - 2019. - Vol. 42. - № 2/W12. - Pp. 167-171.

[19] Raghavendra R., Busch C. Novel image fusion scheme based on dependency measure for robust multispectral palmprint recognition // Pattern recognition. - 2014. - Vol. 47. - №. 6. - Pp. 2205-2221.

[20] Renault C., Desvignes M., Revenu M. 3D curves tracking and its application to cortical sulci detection //Image Processing, 2000. Proceedings. 2000 International Conference on. - IEEE, 2000. - Vol. 2. - Pp. 491-494.

[21] Rosdi B. A., Shing C. W., Suandi S. A. Finger vein recognition using local line binary pattern //Sensors. - 2011. - Vol. 11. - №. 12. - Pp. 11357-11371.

[22] Sun Z., Tan T. Ordinal measures for iris recognition //IEEE Transactions on pattern analysis and machine intelligence. - 2008. - Vol. 31. - №. 12. - Pp. 2211-2226.

[23] Sun Z., Wang L., Tan T. Ordinal feature selection for iris and palmprint recognition//IEEE Transactions on Image Processing. - 2014. - T. 23. - №. 9. - C. 3922-3934.

[24] Thapar D., Jaswal G., Nigam A., Kanhangad V. PVSNet: Palm Vein Authentication Siamese Network Trained using Triplet Loss and Adaptive Hard Mining by Learning Enforced Domain Specific Features - arXiv, 2018.

[25] Wang J., Yang K., Pan Z., Wang G., Li M., Li Y. MinutiaeBased Weighting Aggregation of Deep Convolutional Features for Vein Recognition - IEEE, 2018.

[26] Wang L., Leedham G., Cho D. S. Y. Minutiae feature analysis for infrared hand vein pattern biometrics //Pattern recognition. - 2008. - Vol. 41. - №. 3. - Pp. 920-929.

[27] Wu J. D., Liu C. T. Finger-vein pattern identification using principal component analysis and the neural network technique //Expert Systems with Applications. - 2011. Vol. 38. - №. 5. - Pp. 5423-5427.

[28] Zhou Y., Kumar A. Human identification using palm-vein images //IEEE transactions on information forensics and security. - 2011. - Vol. 6. - №. 4. - Pp. 1259-1274.

[29] Zuiderveld K. Contrast limited adaptive histogram equalization //Graphics gems. - 1994. - Pp. 474-485.

\section{About authors}

Ekaterina I. Safronova, master student at the Lomonosov Moscow State University, Faculty of Computational Mathematics and Cybernetics, Chair of Mathematical Physics. E-mail: katyasafit@gmail.com.

Elena A. Pavelyeva, $\mathrm{PhD}$, assistant professor at the Lomonosov Moscow State University, Faculty of Computational Mathematics and Cybernetics, Chair of Mathematical Physics. E-mail: paveljeva@yandex.ru. 